\title{
MINIREVIEW
}

\section{Host Factors in Positive-Strand RNA Virus Genome Replication}

\author{
Paul Ahlquist, ${ }^{1,2 *}$ Amine O. Noueiry, ${ }^{1}$ Wai-Ming Lee, ${ }^{1,2}$ \\ David B. Kushner, ${ }^{1}$ and Billy T. Dye ${ }^{1,2}$ \\ Institute for Molecular Virology ${ }^{1}$ and Howard Hughes Medical Institute, ${ }^{2}$ \\ University of Wisconsin-Madison, Madison, Wisconsin 53706
}

All viruses are gene poor relative to their hosts: even the largest viral genomes only encode hundreds of genes, while those of host cells typically encode tens of thousands of genes. Thus, most steps in virus infection involve interactions between relatively few different types of viral components and much more complex pools of host factors. This sea of host factors represents both the essential milieu to which viruses must adapt for survival and a tremendous, manipulatable resource for gene-poor viruses. Accordingly, host factors play important roles in most steps of viral infection, and identifying such host factors and their contributions has long been recognized as an important frontier. The continuing emergence of close integration between viral and host functions in infection suggests moving beyond separate views of virus and host to a more holistic view of the virus-infected cell as a unified entity that constitutes the functional unit of infection.

One area in which the importance of host factors is increasingly emerging is the replication of positive-strand RNA viruses. Positive-strand RNA viruses encompass over one-third of all virus genera and include numerous pathogens, such as the severe acute respiratory syndrome coronavirus SARS, hepatitis $\mathrm{C}$ virus (HCV), and many of the viruses on the U.S. Health and Human Services Department Select List of potential bioterrorism agents. Host factors participate in most, if not all, steps of positive-strand RNA virus infection, including entry, viral gene expression, virion assembly, and release. Moreover, host factors are targeted by positive-strand RNA viruses to modulate host gene expression and defenses.

This review focuses on host factors involved in positivestrand RNA virus genome replication. The evidence for such host factor involvement has come from varied genetic and biochemical approaches (15). These include, among others, studies based on the varying permissiveness of some cell types and extracts for RNA replication $(2,3,4,19)$; identification of many host proteins that interact with viral genomic RNAs or replication proteins and, in some cases, have been functionally linked to replication $(5,18,27,35,37,40)$; and mutational screens in genetic model systems, such as Arabidopsis thaliana $(17,39)$ and the yeast Saccharomyces cerevisiae $(8,11,16,23$, 38). Recent data show that host factors play important roles in assembling the viral RNA replication complex, selecting and

\footnotetext{
* Corresponding author. Mailing address: Institute for Molecular Virology, University of Wisconsin-Madison, 1525 Linden Dr., Madison, WI 53706-1596. Phone: (608) 263-5916. Fax: (608) 265-9214. Email: ahlquist@facstaff.wisc.edu.
}

recruiting viral RNA replication templates, activating the complex for RNA synthesis, and other steps. Each of these virushost interactions may contribute to the host specificity, tissue specificity, or pathology of infections. Each such virus-host interaction also represents a potential target for virus control or for optimization to improve beneficial uses of viruses and their components.

Positive-strand RNA viruses can be divided into a number of superfamilies defined by distinguishable RNA replication genes and features (41). Nevertheless, the RNA replication mechanisms of these viruses share sufficient similarities to make it reasonable to discuss their replication as a class and to attempt to draw general lessons by comparing examples from different superfamilies. As discussed further below, a few of the common features shared by positive-strand RNA viruses are the need to coordinate use of the infecting viral genomic RNA as a template for translation and replication, assembly of replication complexes on intracellular membranes, and production of 10- to 100 -fold excesses of positive- over negativestrand RNA.

A simplified general scheme for RNA replication by positive-strand RNA viruses is shown in Fig. 1. Below we discuss the involvement of host factors in the various stages of this RNA replication process, using examples from a number of viruses. We regret that space limitations do not allow us to cite all of the work being done in this exciting area.

\section{TRANSLATION AND RNA REPLICATION}

Positive-strand RNA virus genomes are templates for both translation and replication, leading to interactions between host translation factors and RNA replication at multiple levels. All known positive-strand RNA viruses carry genes for an RNA-dependent RNA polymerase ( $\mathrm{RdRp}$ ) used in genome replication. However, unlike other RNA viruses, positivestrand RNA viruses do not encapsidate this polymerase. Thus, upon infection of a new cell, viral RNA replication cannot begin until the genomic RNA is translated to produce polymerase and, for most positive-strand RNA viruses, additional replication factors involved in membrane targeting, template recruitment, RNA capping, and other functions. Moreover, RNA replication by poliovirus and some other viruses requires translation in cis, providing a partial check on genome functionality (25). Host factors involved in virus translation and viral takeover of translation have been reviewed elsewhere (e.g., see references 6 and 12). 


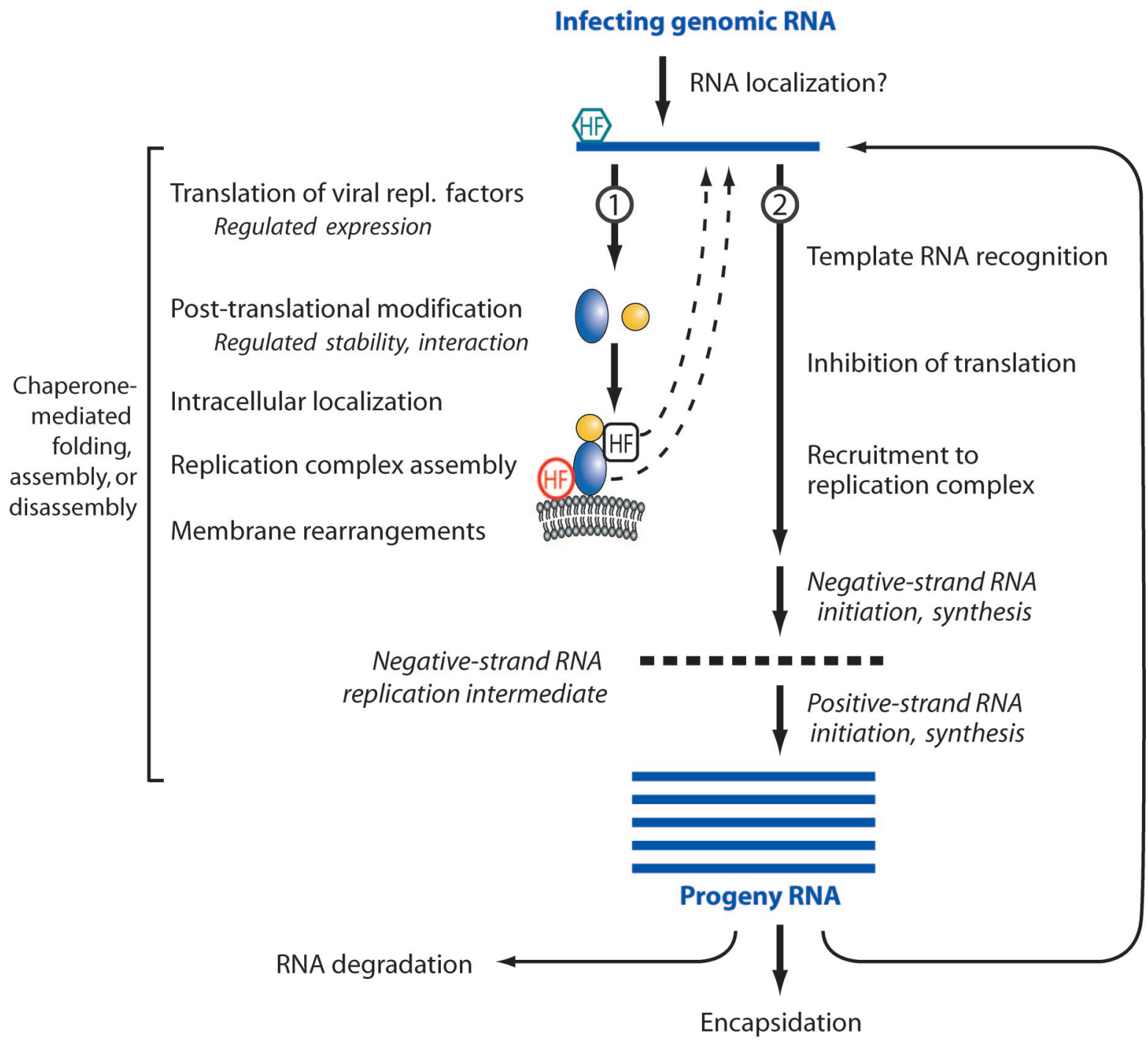

FIG. 1. Highly simplified schematic of major steps in positive-strand RNA virus genome replication. Arrows 1 and 2 depict the sequential use of the infecting, positive-strand genomic RNA as a template first for translation and then for RNA replication. As discussed in the text, precedents exist for host factor involvement in most steps shown. Based on examples cited in this review, sample host factors (HF) are shown interacting with viral RNA, viral RNA replication proteins, and host membranes. Some host factors may be associated with viral components only transiently during particular steps of the replication process.

Host factors can mediate viral translation generally or can be involved in selective translational regulation of specific viral factors in ways important for RNA replication. Brome mosaic virus (BMV), a member of the alphavirus-like superfamily of positive-strand RNA viruses, directs RNA replication, subgenomic mRNA transcription, and virion assembly in yeast. Yeast genetic approaches have identified a number of host genes required for BMV RNA replication (11). One such host gene, $D E D 1$, encodes an RNA helicase required for translation initiation on all yeast mRNAs. Although $D E D 1$ is an essential gene, certain mutations in $D E D 1$ block BMV RNA replication without inhibiting cell growth (23). This block is associated with selective loss of BMV polymerase translation and is tightly linked with 5 '-proximal, cis-acting sequences that downregulate polymerase translation relative to a second, helicase-like RNA replication factor translated from a separate BMV ge- nomic RNA. Similar downregulation of polymerase levels relative to other replication factors is important for efficient replication by many other positive-strand RNA viruses and retroviruses but is more commonly achieved by translational frameshift or readthrough mechanisms rather than at translation initiation $(5,35)$. Further similarities of positive-strand RNA viruses to retroviruses are noted below. In addition to translational regulation, some positive-strand RNA viruses use host turnover pathways to regulate the relative accumulation of viral RNA replication factors (35).

Although the viral genomic RNA must first be translated, 5'-to-3' ribosome trafficking blocks $3^{\prime}$-to-5' polymerase copying of viral RNA (2). Thus, after initial replication factor synthesis, positive-strand RNA viruses must switch the template role of the incoming genomic RNA from translation to replication. Poliovirus translation and negative-strand RNA synthe- 
sis are coordinated by the interaction of a complex of host and viral factors with each other and with both ends of the viral RNA, including a multifunctional 5' cloverleaf structure (2, 10, 43). This complex includes host $\operatorname{poly}(\mathrm{C})$ and $\operatorname{poly}(\mathrm{A})$ binding proteins (PCBP and PABP, respectively) and viral polymerase precursor $3 C D$. Early in infection, interaction of PCBP and PABP with the 5' and $3^{\prime}$ RNA ends, respectively, and with each other promotes translation. Later in infection, when $3 \mathrm{CD}$ accumulates, 3CD interaction with the 5 ' cloverleaf and associated PCBP-PABP-RNA complex inhibits translation and promotes initiation of negative-strand RNA synthesis at the $3^{\prime}$ end of the RNA. The $3^{\prime}$ noncoding region of poliovirus RNA also binds nucleolin, another host RNA binding protein, and immunodepletion of nucleolin inhibits early yields of poliovirus virions in a cell-free replication system (42). Thus, nucleolin may facilitate one or more interactions of poliovirus RNA during early infection when viral RNA or protein levels are low. During poliovirus infection, nucleolin and some other host proteins implicated in virus replication undergo significant relocalization from the nucleus to the cytoplasm $(20,42)$.

Interactions between viral RNA, general host translation factors, and RNA binding proteins may be common in the picornavirus-like superfamily, since the polymerase of another member in the plant-infecting potyvirus family interacts with PABP (44). In addition, the $5^{\prime}$ genome-linked proteins of some potyviruses bind to cellular cap-binding proteins eIF4E or eIF(iso)4E, and eIF(iso)4E mutations block replication by one potyvirus (17). Several possible functions in translation or replication have been proposed for this interaction.

In a possible parallel with poliovirus 3CD-RNA interaction, BMV genomic RNAs are recruited from translation to replication by viral protein 1a, a helicase-like replication factor, which acts through cis-acting recognition elements (REs) on each viral RNA. Like the $5^{\prime}$ poliovirus RNA cloverleaf, the REs on BMV genomic RNAs 1 and 2 are located at the 5' end of the RNA, while BMV RNA3 contains an internal RE that appears to interact with its $5^{\prime}$ end $(7,8)$. Efficient 1 a recruitment of viral RNAs also requires a host gene, LSM1 (8). LSM1 encodes a protein with the conserved Sm motif, which associates with other Sm motif proteins in complexes involved in mRNA turnover and other processes. In a further parallel to coordination of poliovirus translation and replication, recent results show that LSM1 and the Sm complex involved in cellular mRNA turnover are also required for translation of BMV genomic RNAs destined for replication, linking BMV RNA translation and replication (24). Interestingly, initiation of RNA synthesis by positive-strand RNA bacteriophage $Q \beta$ also involves a host $\mathrm{Sm}$ protein, $\mathrm{Hfq}$, which regulates turnover of some bacterial mRNAs (34). In addition to 1a and LSM1, BMV RNA recruitment may involve host factors that interact with tRNAs, since the REs contain the conserved sequence and structure elements of the T $\Psi \mathrm{C}$ stem-loops of host tRNAs, and these functions are essential to the $1 \mathrm{a}$ responsiveness of the REs (7).

Other connections with translation are found in the association of translation factors with active viral RNA replication complexes. Subunits of host translation initiation factor eIF3 are associated with virus-specific RdRp-complexes isolated from cells infected by BMV or tobacco mosaic virus (TMV). A 41-kDa subunit of eIF3 copurified with BMV RdRp and in- teracted with BMV 2a polymerase, and addition of purified eIF3 or the 41-kDa subunit stimulated negative-strand synthesis by BMV RdRp (28). TMV RNA replication proteins interacted with and coimmunoprecipitated with the host GCD10 subunit of eIF3, and anti-GCD10 antibodies inhibited negative-strand synthesis by TMV RdRp $(26,36)$. Such host factors may have functions related to the aminoacylatable $3^{\prime}$ tRNAlike elements of TMV and BMV genomic RNAs (where negative-strand RNA synthesis initiates), to the tRNA TYC stemloops of the BMV RE template-recruitment signals, or to both.

Before and after replication, viral RNA requires protection from cellular RNA degradation pathways including potent 5' and $3^{\prime}$ exonucleases. Cellular mRNAs and many viral RNAs are protected against these turnover pathways by cellular $5^{\prime}$ cap binding proteins and $3^{\prime}$ PABPs. The stability of poliovirus RNA, which lacks a 5' cap, is promoted by host PCBP binding to the 5' cloverleaf (22). Conversely, many viral RNAs lack a 3' poly(A) but have $3^{\prime}$-terminal secondary structures that bind host proteins, which may contribute to protecting the RNA from $3^{\prime}$ exonucleases (references 5 and 18 and references therein). As noted above, the RNAs of BMV and some other positive-strand RNA viruses have multifunctional, 3' tRNAlike structures. These 3' tRNA-like structures bind translation initiation factors and are matured by host tRNA nucleotidyltransferase, which adds untemplated nucleotides to BMV RNAs and thus performs a telomerase-like function to maintain the viral RNA 3' ends (5). The resulting tRNA-like $\mathrm{CCA}_{\mathrm{OH}} 3^{\prime}$ ends of the viral RNAs are substrates in vivo for host aminoacyl tRNA synthetases, which esterify a specific amino acid (tyrosine for BMV) to the $3^{\prime}$ hydroxyl as if charging a tRNA.

\section{ASSEMBLY OF MEMBRANE-ASSOCIATED RNA REPLICATION COMPLEXES}

All characterized positive-strand RNA viruses assemble their RNA replication complexes on intracellular membranes, usually in association with membrane vesicle formation or other membrane rearrangements. For example, RNA replication by many viruses in and beyond the alphavirus-like superfamily occurs in association with 50- to 70-nm spherical invaginations of intracellular membranes. Recent results with one such virus, BMV, revealed that the structure, assembly, and function of such replication complexes share multiple parallels with retrovirus and double-stranded RNA (dsRNA) virus cores (31). Specifically, the helicase-like BMV 1a protein mimics the roles of Gag protein in retrovirus capsid assembly by inducing formation of the spherical membrane invaginations and selectively packaging the viral genomic RNAs. Similar to a budding, membrane-enveloped capsid, each resulting spherule invaginates or partially buds into the endoplasmic reticulum (ER) lumen and contains hundreds of 1a proteins. The cisacting BMV REs function as virus-specific RNA packaging signals, paralleling retroviral RNA packaging signals. If BMV 2a polymerase is coexpressed, a few copies of $2 \mathrm{a}$ are incorporated into each of the capsid-like spherules, which then become the sites of viral negative- and positive-strand RNA synthesis. The final replication complex contains a $1 \mathrm{a} / 2 \mathrm{a}$ polymerase ratio of approximately 25 , similar to the Gag/GagPol ratio of retrovirus capsids. 
For such replication complexes, the host membrane constitutes a crucial host factor serving multiple purposes. First, the membrane provides a surface on which replication factors are localized and concentrated for assembly. The membrane also surrounds and protects the virus-induced replication compartment, similar to a budding virion, in which the RNA replication factors and genomic RNAs are sequestered from competing RNA templates and competing processes such as translation. This organization also helps to protect any dsRNA replication intermediates from dsRNA-induced host defense responses such as RNA interference or interferon-induced responses (1). The RNA replication complex thus constitutes a new, membrane-bounded compartment with specific components and functions and so could be considered a virus-specific organelle for RNA replication.

While many positive-strand RNA viruses form similar spherical invaginations, others form distinct membrane structures, including alternate types of vesicles or appressed membranes (references 30 and 31 and references therein). Such alternate membrane rearrangements may serve similar functions in concentrating and sequestering viral RNA replication factors and templates and, though their detailed organization is not yet defined, might be topologically related to the spherical replication complexes.

Various viruses assemble their RNA replication complexes on different, but usually specific, membranes or membrane subsets. For example, alphaviruses use endosomal and lysosomal membranes (13), BMV uses ER membranes (29), nodaviruses use mitochondrial membranes (21), and poliovirus replication complexes are formed on densely packed vesicles implicated to be derived from the cellular COPII transport vesicle budding pathway (30). While such specific membrane localization must involve interaction with host factors, many details of such targeting remain incompletely understood.

For many viral replication proteins, localization to such membranes depends on interaction with another viral protein. Alphavirus nsP1, for example, directs nsP2, -3, and -4 to endosomal membranes (13), and HCV NS4A localizes the viral NS3 protease-helicase to the perinuclear ER (37). Similarly, BMV 1a protein interacts with and directs the 2 a polymerase to ER membranes (7). For the related cucumber mosaic virus, $1 \mathrm{a}-2 \mathrm{a}$ interaction is disrupted by $2 \mathrm{a}$ phosphorylation by a host kinase (14). Such 2a phosphorylation occurs in vivo and may inhibit formation of new replication complexes late in infection (14) or may help to maintain the approximately $25: 1$ ratio of $1 \mathrm{a}$ to $2 \mathrm{a}$ in replication complexes (31). The observed host phosphorylation of RNA replication proteins in other viruses, such as alphavirus nsP3 (13), HCV NS5A (37), and others, may similarly contribute to regulating replication.

For at least some viruses, host membrane proteins may be temporary or permanent components of the RNA replication complex and, in addition to other functions, may be involved in loading or retaining viral replication factors on membranes $(9$, 45). Notably, TMV replication in Arabidopsis is blocked by dual mutation of host integral membrane protein TOM1 and its homolog TOM3. TOM1 interacts with the shared helicaselike domain of the overlapping TMV 130- and 180-kDa RNA replication factors and cofractionates with TMV replication proteins and RdRp activity. TOM1 also interacts with
TOM2A, another host integral membrane protein required for efficient TMV replication.

\section{VIRAL RNA SYNTHESIS}

After viral RNA replication factors and templates are assembled into a complex on host membranes, at least some viruses require additional contributions from host factors to initiate RNA synthesis. Preformed, sedimentable poliovirus RNA replication complexes, when isolated before RNA initiation by reversible inhibition with guanidine, require the addition of soluble cytoplasmic factors from uninfected cells to become competent for viral RNA synthesis (3). Similarly, replication of poliovirus RNA in Xenopus laevis oocytes requires coinjection of human cell extracts for a posttranslation step, although it is not known whether the same step or a different step is affected (2).

Similarly, BMV RNA replication is blocked after initial replication complex assembly by mutations in the yeast gene YDJ1 (38). Cells bearing such YDJ1 mutations support many early steps in RNA replication at normal levels, including association of the viral 1a protein with membranes, 1a-mediated recruitment and stabilization of viral RNA replication templates, and 1a-mediated recruitment of the viral $2 \mathrm{a}$ polymerase. However, YDJ1 mutant cells do not support initiation of negativestrand RNA synthesis. YDJ1 encodes a chaperone that is related to Escherichia coli DnaJ and is partially localized to ER membranes, the site of BMV RNA synthesis. Moreover, in YDJ1 mutant yeast but not wild-type yeast, a large fraction of 2a polymerase was found in a membrane-free but rapidly sedimenting form, suggesting aggregation. Thus, the YDJ1 chaperone function may direct a 2 a polymerase folding or interaction step that is required for RNA synthesis. Host chaperones are also involved in proteolytic processing of $\mathrm{HCV}$ nonstructural proteins (37).

Mutations in another host gene, OLE1, also block BMV RNA replication after membrane association of 1a, 2a polymerase, and viral RNA but before initiation of negative-strand RNA synthesis (16). OLE1 is an essential gene encoding $\Delta 9$ fatty acid desaturase, the key enzyme for converting saturated to unsaturated fatty acids. Although this enzyme is an integral ER membrane protein and thus is localized to the site of BMV RNA replication, medium supplementation experiments show that BMV RNA synthesis does not require the OLE1-encoded protein but rather is much more sensitive than cell growth to reductions in unsaturated fatty acid levels. This high sensitivity to membrane lipid composition dramatically illustrates the functional importance of membrane association for RNA replication. The need for high unsaturated fatty acid levels also suggests that the replication complex may require significant membrane fluidity or plasticity and thus may be in a dynamic relationship with the host membrane.

Host factors thus may contribute to initiation of negativestrand RNA synthesis in many ways, including inhibiting viral RNA translation, activating specific functions of the replication complex, and recognizing and utilizing the $3^{\prime}$ end of positive-strand RNA as an initiation site $(5,15,42)$. Host factors may also be involved in initiation of positive-strand RNA synthesis from negative-strand RNA templates. Poliovirus positive-strand RNA synthesis requires base pairing of the 5' clo- 
verleaf structure in the nontemplate, positive-strand RNA. One proposed explanation is that the negative-strand template RNA may be bound in a dsRNA replicative form. Initiation of new positive-strand RNA would then require unpairing of the $3^{\prime}$ end of the negative-strand RNA template from this dsRNA form, which could be facilitated by the complementary $5^{\prime}$ end of positive-strand RNA forming the cloverleaf secondary structure, which then is stabilized by binding host PCBP and viral proteins (2, 27; also, see above).

For the flavivirus West Nile virus, a 3'-terminal stem-loop on negative-strand RNA binds several host proteins, including TIAR and its homolog TIA-1, host RNA binding proteins with roles in translation and RNA splicing (18). West Nile virus growth was significantly reduced in murine cell lines lacking TIAR. Since the only known role of this negative-strand RNA is to act as a replication intermediate, TIAR may contribute to positive-strand RNA synthesis. For coronaviruses, binding of host RNA binding factor hnRNP A1 to strategic sites in viral negative- and positive-strand RNA may be involved in subgenomic mRNA transcription and RNA replication, although the role of this protein in vivo has been debated $(32,33)$.

\section{CONCLUDING REMARKS}

In summary, emerging results indicate that positive-strand RNA virus genome replication and associated infection events depend on a wide range of host factors and that these factors contribute a varied array of functions to the assembly and function of the viral replication complex and the fate of its product RNAs. While significant progress has been made, crucial details remain to be elucidated for many important steps, such as the mechanisms targeting replication proteins and templates to specific intracellular membranes. Thus, present results likely have revealed only a small fraction of the underlying virus-host interactions on which replication depends. Progress in these areas should continue to accelerate due to many advances, including increasing synergy between emerging results, further definition of protein interaction maps, and availability of RNA interference approaches and other improved tools to test the roles of specific gene products. Past and recent experience assures that the results not only will contribute to understanding and control of virus-specific processes but also will advance understanding of many normal cell pathways of broad relevance to biology and medicine.

\section{ACKNOWLEDGMENTS}

We thank Ann Palmenberg, Bill Sugden, and many other colleagues for valuable discussions and insights.

This work was supported by National Institutes of Health grant GM35072. P.A. is an investigator of the Howard Hughes Medical Institute.

\section{REFERENCES}

1. Ahlquist, P. 2002. RNA-dependent RNA polymerases, viruses, and RNA silencing. Science 296:1270-1273.

2. Andino, R., N. Boddeker, D. Silvera, and A. V. Gamarnik. 1999. Intracellular determinants of picornavirus replication. Trends Microbiol. 7:76-82.

3. Barton, D. J., E. P. Black, and J. B. Flanegan. 1995. Complete replication of poliovirus in vitro: preinitiation RNA replication complexes require soluble cellular factors for the synthesis of VPg-linked RNA. J. Virol. 69:5516-5527.

4. Blight, K. J., A. A. Kolykhalov, and C. M. Rice. 2000. Efficient initiation of HCV RNA replication in cell culture. Science 290:1972-1974.

5. Buck, K. W. 1996. Comparison of the replication of positive-stranded RNA viruses of plants and animals. Adv. Virus Res. 47:159-251.
6. Bushell, M., and P. Sarnow. 2002. Hijacking the translation apparatus by RNA viruses. J. Cell Biol. 158:395-399.

7. Chen, J., A. Noueiry, and P. Ahlquist. 2001. Brome mosaic virus protein 1a recruits viral RNA2 to RNA replication through a 5' proximal RNA2 signal. J. Virol. 75:3207-3219.

8. Diez, J., M. Ishikawa, M. Kaido, and P. Ahlquist. 2000. Identification and characterization of a host protein required for efficient template selection in viral RNA replication. Proc. Natl. Acad. Sci. USA 97:3913-3918.

9. Hagiwara, Y., K. Komoda, T. Yamanaka, A. Tamai, T. Meshi, R. Funada, T. Tsuchiya, S. Naito, and M. Ishikawa. 2003. Subcellular localization of host and viral proteins associated with tobamovirus RNA replication. EMBO J. 22:344-353.

10. Herold, J., and R. Andino. 2001. Poliovirus RNA replication requires genome circularization through a protein-protein bridge. Mol. Cell 7:581591.

11. Ishikawa, M., J. Diez, M. Restrepo-Hartwig, and P. Ahlquist. 1997. Yeast mutations in multiple complementation groups inhibit brome mosaic virus RNA replication and transcription and perturb regulated expression of the viral polymerase-like gene. Proc. Natl. Acad. Sci. USA 94:13810-13815.

12. Jackson, R. J. 2002. Proteins involved in the function of picornavirus internal ribosomal entry sites, p. 171-183. In B. L. Semler and E. Wimmer (ed.), Molecular biology of picornaviruses. ASM Press, Washington, D.C.

13. Kaariainen, L., and T. Ahola. 2002. Functions of alphavirus nonstructural proteins in RNA replication. Prog. Nucleic Acid Res. Mol. Biol. 71:187-222.

14. Kim, S. H., P. Palukaitis, and Y. I. Park. 2002. Phosphorylation of cucumber mosaic virus RNA polymerase 2a protein inhibits formation of replicase complex. EMBO J. 21:2292-2300.

15. Lai, M. M. 1998. Cellular factors in the transcription and replication of viral RNA genomes: a parallel to DNA-dependent RNA transcription. Virology 244:1-12.

16. Lee, W. M., M. Ishikawa, and P. Ahlquist. 2001. Mutation of host $\Delta 9$ fatty acid desaturase inhibits brome mosaic virus RNA replication between template recognition and RNA synthesis. J. Virol. 75:2097-2106.

17. Lellis, A. D., K. D. Kasschau, S. A. Whitham, and J. C. Carrington. 2002. Loss-of-susceptibility mutants of Arabidopsis thaliana reveal an essential role for eIF(iso)4E during potyvirus infection. Curr. Biol. 12:1046-1051.

18. Li, W., Y. Li, N. Kedersha, P. Anderson, M. Emara, K. M. Swiderek, G. T. Moreno, and M. A. Brinton. 2002. Cell proteins TIA-1 and TIAR interact with the $3^{\prime}$ stem-loop of the West Nile virus complementary minus-strand RNA and facilitate virus replication. J. Virol. 76:11989-12000.

19. Lohmann, V., S. Hoffmann, U. Herian, F. Penin, and R. Bartenschlager. 2003. Viral and cellular determinants of hepatitis C virus RNA replication in cell culture. J. Virol. 77:3007-3019.

20. McBride, A. E., A. Schlegel, and K. Kirkegaard. 1996. Human protein Sam68 relocalization and interaction with poliovirus RNA polymerase in infected cells. Proc. Natl. Acad. Sci. USA 93:2296-2301.

21. Miller, D. J., M. D. Schwartz, and P. Ahlquist. 2001. Flock house virus RNA replicates on outer mitochondrial membranes in Drosophila cells. J. Virol. 75:11664-11676.

22. Murray, K. E., A. W. Roberts, and D. J. Barton. 2001. Poly(rC) binding proteins mediate poliovirus mRNA stability. RNA 7:1126-1141.

23. Noueiry, A. O., J. Chen, and P. Ahlquist. 2000. A mutant allele of essential, general translation initiation factor DED1 selectively inhibits translation of a viral mRNA. Proc. Natl. Acad. Sci. USA 97:12985-12990.

24. Noueiry, A. O., J. Diez, S. Falk, J. Chen, and P. Ahlquist. 2003. Yeast Lsm1p-7p/Pat1p deadenylation-dependent mRNA decapping factors are required for brome mosaic virus genomic RNA translation. Mol. Cell. Biol. 23:4094-4106.

25. Novak, J. E., and K. Kirkegaard. 1994. Coupling between genome translation and replication in an RNA virus. Genes Dev. 8:1726-1737.

26. Osman, T. A., and K. W. Buck. 1997. The tobacco mosaic virus RNA polymerase complex contains a plant protein related to the RNA-binding subunit of yeast eIF-3. J. Virol. 71:6075-6082.

27. Paul, A. V. 2002. Possible unifying mechanism of picornavirus genome replication, p. 227-246. In B. L. Semler and E. Wimmer (ed.), Molecular biology of picornaviruses. ASM Press, Washington, D.C

28. Quadt, R., C. C. Kao, K. S. Browning, R. P. Hershberger, and P. Ahlquist. 1993 Characterization of a host protein associated with brome mosaic virus RNAdependent RNA polymerase. Proc. Natl. Acad. Sci. USA 90:1498-1502.

29. Restrepo-Hartwig, M. A., and P. Ahlquist. 1996. Brome mosaic virus helicase- and polymerase-like proteins colocalize on the endoplasmic reticulum at sites of viral RNA synthesis. J. Virol. 70:8908-8916.

30. Rust, R. C., L. Landmann, R. Gosert, B. L. Tang, W. Hong, H. P. Hauri, D. Egger, and K. Bienz. 2001. Cellular COPII proteins are involved in production of the vesicles that form the poliovirus replication complex. J. Virol. 75:9808-9818.

31. Schwartz, M., J. Chen, M. Janda, M. Sullivan, J. den Boon, and P. Ahlquist. 2002. A positive-strand RNA virus replication complex parallels form and function of retrovirus capsids. Mol. Cell 9:505-514.

32. Shen, X., and P. S. Masters. 2001. Evaluation of the role of heterogeneous nuclear ribonucleoprotein A1 as a host factor in murine coronavirus discon- 
tinuous transcription and genome replication. Proc. Natl. Acad. Sci. USA 98:2717-2722

33. Shi, S. T., P. Huang, H. P. Li, and M. M. Lai. 2000. Heterogeneous nuclear ribonucleoprotein A1 regulates RNA synthesis of a cytoplasmic virus. EMBO J. 19:4701-4711.

34. Sledjeski, D. D., C. Whitman, and A. Zhang. 2001. Hfq is necessary for regulation by the untranslated RNA DsrA. J. Bacteriol. 183:1997-2005.

35. Strauss, J. H., and E. G. Strauss. 1994. The alphaviruses: gene expression, replication, and evolution. Microbiol. Rev. 58:491-562.

36. Taylor, D. N., and J. P. Carr. 2000. The GCD10 subunit of yeast eIF-3 binds the methyltransferase-like domain of the 126 and $183 \mathrm{kDa}$ replicase proteins of tobacco mosaic virus in the yeast two-hybrid system. J. Gen. Virol. 81: $1587-1591$.

37. Tellinghuisen, T. L., and C. M. Rice. 2002. Interaction between hepatitis C virus proteins and host cell factors. Curr. Opin. Microbiol. 5:419-427.

38. Tomita, Y., T. Mizuno, J. Diez, S. Naito, P. Ahlquist, and M. Ishikawa. 2003 Mutation of host dnaJ homolog inhibits brome mosaic virus negative-strand RNA synthesis. J. Virol. 77:2990-2997.

39. Tsujimoto, Y., T. Numaga, K. Ohshima, M. A. Yano, R. Ohsawa, D. Goto, S Naito, and M. Ishikawa. 2003. Arabidopsis tobamovirus multiplication
(TOM) 2 locus encodes a transmembrane protein that interacts with TOM1. EMBO J. 22:335-343.

40. van der Heijden, M. W., and J. F. Bol. 2002. Composition of alphavirus-like replication complexes: involvement of virus and host encoded proteins. Arch. Virol. 147:875-898.

41. van Regenmortel, M. H. V. (ed.). 2000. Virus taxonomy. Academic Press, San Diego, Calif.

42. Waggoner, S., and P. Sarnow. 1998. Viral ribonucleoprotein complex for mation and nucleolar-cytoplasmic relocalization of nucleolin in poliovirusinfected cells. J. Virol. 72:6699-6709.

43. Walter, B. L., T. B. Parsley, E. Ehrenfeld, and B. L. Semler. 2002. Distinct poly $(\mathrm{rC})$ binding protein $\mathrm{KH}$ domain determinants for poliovirus translation initiation and viral RNA replication. J. Virol. 76:12008-12022.

44. Wang, X., Z. Ullah, and R. Grumet. 2000. Interaction between zucchini yellow mosaic potyvirus RNA-dependent RNA polymerase and host poly(A) binding protein. Virology 275:433-443.

45. Yamanaka, T., T. Ohta, M. Takahashi, T. Meshi, R. Schmidt, C. Dean, S. Naito, and M. Ishikawa. 2000. TOM1, an Arabidopsis gene required for efficient multiplication of a tobamovirus, encodes a putative transmembrane protein. Proc. Natl. Acad. Sci. USA 97:10107-10112. 